\title{
Е.Н. Малюга
}

\section{НОВЫЕ ТЕНДЕНЦИИ АНГЛОЯЗЫЧНОГО НАУЧНОГО ДИСКУРСА: ВОПРОСЫ АКТУАЛЬНОСТИ ИССЛЕДОВАНИЯ И ЯЗЫКОВОЙ ИДЕНТИЧНОСТИ ${ }^{1}$}

\begin{abstract}
Исследуется формат англоязычных научных публикаций, отмеченных сниженной актуальностью темы исследования, которая контрастирует с методологической и научно-исследовательской иелесообразностью научного текста в целом, создавая эффект парадокса. Анализируются функциональные, языковые и дискурсивные особенности научных публикаций в рамках современного англоязычного научного дискурса. Рассматриваются соответствующие предпосылки к изучению идентичности автора как создателя научного текста.

Ключевые слова: научный дискурс, идентичность, научная публикачия, научная статья, актуальность исследования, дискурсивный маркер, идентифиичирующая функция, прецедент.
\end{abstract}

В статье исследуется научный дискурс, представляющий собой письменное или устное речетворчество в сфере науки, отличающееся «познавательной направленностью, информативностью, аргументированностью, высокой логической культурой, а также интеллектуальной прогрессивностью и эстетической оформленностью» [1. С. 11]. Выступая участниками научного дискурса, исследователи взаимодействуют друг с другом «в соответствии с нормами, установленными научным сообществом» [2. С. 131], используя средства языка с целью обозначения и описания научной проблемы. При этом центральным параметром любого научного текста традиционно выступает актуальность исследования, указывающая на «необходимость и своевременность изучения и решения проблемы для дальнейшего развития теории и практики» [3. С. 6]. Актуальность исследования определяет и общую содержательную значимость научной работы, призванной внести вклад в решение конкретной проблемы, релевантной для человечества в целом и для научного сообщества в частности.

В настоящее время стремительное развитие научного дискурса порождает новые формы представления знания и научного доказательства, и одним из наиболее неконвенциональных примеров нового формата научной риторики является формат, в рамках которого теме исследования намеренно отводится периферийная роль, в то время как методология исследования выходит на передний план, становясь, по сути, самоцелью научной

\footnotetext{
${ }^{1}$ Публикация подготовлена при поддержке Программы РУДН «5-100».
} 
работы в целом. Одним из ярких примеров, иллюстрирующих сущность рассматриваемого явления, служит статья, опубликованная в журнале «Economic Inquiry» под заголовком «On the efficiency of AC/DC: Bon Scott versus Brian Johnson» [4]. Содержание статьи раскрывается авторами в аннотации следующим образом:

We use tools from experimental economics to address the age-old debate regarding who was a better singer in the band AC/DC. Our results suggest that (using wealth maximization as a measure of "better») listening to Brian Johnson (relative to listening to Bon Scott) resulted in "better» outcomes in an ultimatum game. These results may have important implications for settling drunken music debates and environmental design issues in organizations [Ibid. P. 598].

Mbl используем экспериментальные методы экономического анализа с ичелью разрешения давнего спора о том, кто является лучшим певцом в группе $A C / D C$. Проведенное исследование подтверждает, что (с учетом применения теории максимизации стоимости в качестве мерила качества) прослушивание песен Брайана Джсосона (в сравнении с прослушиванием песен Бона Скотта) оказывается более эффективным при проведении экспериментальной игры «ультиматум». Эти результаты могут получить практическое применение при разрешении музыкальных споров во время распития спиртных напитков, а также при решении вопросов природоохранного проектирования на производствах ${ }^{1}$.

Очевидно, что научное исследование, состоящее в эконометрическом измерении субъективных музыкальных предпочтений с целью «разрешения музыкальных споров во время распития спиртных напитков», по меньшей мере, противоречиво. Тем не менее методология анализа, которой авторы придерживаются на протяжении статьи, соответствует всем канонам серьезного экономического исследования, основанного на применении легитимных экономических методов научного познания. Таким образом, тема исследования предстает в качестве своего рода «оболочки», поверхностного бессодержательного «слоя», намеренно лишенного какой-либо практической или теоретической ценности. Периферийный статус темы исследования, в свою очередь, отчетливо контрастирует с неопровержимой актуальностью его методологии, порождая новый продукт научной мысли, центральная задача которого состоит в своего рода «шаблонном» описании процедуры исследования и привлечении внимания читателя к существующим способам научного анализа. Подобные статьи нестандартного формата сегодня публикуются и распространяются влиятельными научными изданиями наравне с работами, написанными в соответствии с традиционными канонами научного письменного дискурса.

С учетом сказанного рассматриваемое явление анализируется в настоящем исследовании в качестве специфического формата построения научно значимого аргумента в англоязычных научных публикациях, двумя взаимосвязанными и четко контрастирующими компонентами которого вы-

\footnotetext{
1 Здесь и далее перевод автора.
} 
ступают «периферийная» актуальность темы исследования и приоритетная актуальность его методологии. Анализируя указанный формат научной аргументации, мы ставим ключевой целью дискурсивный анализ данного явления в англоязычном научном дискурсе. Материалом исследования выступили англоязычные научные публикации экономической тематики, опубликованные в высокорейтинговых рецензируемых научных изданиях:

- «Economic Inquiry» (Великобритания, h-index 61, Scopus Q1/Q2);

- «Applied Economic Perspectives and Policy» (Великобритания, h-index 40, Scopus Q1/Q2);

- «American Economic Review» (СШA, h-index 237, Scopus Q1);

- «Accounting, Organizations and Society» (Великобритания, h-index 110, Scopus Q1);

- «Policy Sciences» (Нидерланды, h-index 45, Scopus Q1);

- «Journal of Political Economy» (США, h-index 159, Scopus Q1); и

- «Journal of Environmental Economics and Management» (CШA, h-index 101, Scopus Q1).

Включение в материал исследования статей, опубликованных в высокорейтинговых научных журналах, позволило обеспечить качество выборки, поскольку высокий рейтинг журнала исключает вероятность допуска к публикации «околонаучных» исследований, не соответствующих строгим правилам жесткой редакционной политики.

Обращение к научным публикациям экономической тематики продиктовано стремлением сузить область исследования путем ограничения анализа рамками отдельного институционального дискурса.

Научные статьи анализируются в данном исследовании в качестве одного из ключевых жанров научного дискурса, обеспечивающего обмен научной информацией. Жанровый анализ позволяет сузить область исследования до отдельных жанров, функционирующих в рамках данного типа дискурса и сформированных в соответствии со сложившейся традицией письменного и устного научного речетворчества, и представляет собой специализированную форму дискурс-анализа, когда внимание исследователя обращено на отдельные повторяющиеся элементы конструирования мысли, включая структурную композицию, грамматику и лексику. При этом в рамках жанрового анализа текст рассматривается как своего рода «ключ», позволяющий исследователю описать не только особенности широкого спектра лингвистических практик, но и специфику людей (в случае научного дискурса - ученых) и профессиональных групп (научного сообщества), участвующих в продуцировании соответствующего типа дискурса. Каждая научная статья как жанр научного дискурса может рассматриваться в качестве отдельного этапа масштабной дискуссии, как очередной аргумент или «одна из реплик в диалоге» [5. С. 17], позволяющая приблизиться к решению конкретной научной проблемы.

Задача любой научной статьи состоит в том, чтобы убедить читателя в корректности и актуальности предоставляемой информации. Для достижения данной цели авторы используют устоявшийся набор языковых средств 
и следуют общепринятым композиционным правилам, оправдывая, таким образом, ожидания читателя, сформированные устойчивой традицией построения научно обоснованных рассуждений. Другими словами, научная статья как жанр научного дискурса предстает в качестве своего рода «договора» между автором и читателем, где обе стороны «обязуются» создавать и интерпретировать информацию, основываясь на правилах, регулирующих процессы продуцирования и осмысления научного текста.

Важной особенностью современной научной статьи становится смещение акцентов, которое подразумевает увеличение значимости дискурсивной состоятельности аргумента, его убедительной формулировки. В то же время текст не может рассматриваться как достоверное отражение реального мира, поскольку любая трактовка является результатом фильтруемой проекции знания, основанной на приоритизации, проистекающей из интенциональных предпосылок исследователя [6]. Другими словами, любой набор данных, выведенных даже через объективно-доказательные методологии анализа, может быть интерпретирован по-разному, и если «вес» конечного аргумента зависит от выбранной автором «траектории» рассуждения, то именно дискурсивные способы аргументирования становятся критическим фактором успешности исследования. С такой точки зрения, поиск доказательной базы при анализе продукта научной деятельности будет зависеть от точности интерпретации дискурсивных практик, используемых автором с целью предупреждения критики в отношении выдвинутых им гипотез и заключений.

Как следствие, интерес к изучению научных статей во многом обусловлен интересом к специфичным способам их оформления, позволяющим предоставить убедительные доказательства выдвигаемых теорий. Исследователи научного дискурса стремятся выяснить, как именно дискурс используется авторами для изложения мысли и конструирования знания (см., например, [7-13] и др.). Это - ключевая проблема в рамках анализа жанровых разновидностей научного дискурса в частности и коммуникации в целом.

Выбор в качестве объекта исследования именно англоязычного научного дискурса обусловливается возможностью обеспечения более обширной выборки, что связано с давно наметившимся приоритетом английского как главного языка письменной научной коммуникации. Некоторые исследователи рассматривают такую тенденцию в качестве проблемы, требующей решения. Например, Р. Менегини и А. Пэкер в статье «Is there science beyond English?» констатируют, что «любой ученый, желающий иметь доступ к актуальным исследованиям и получить международное признание, должен овладеть английским языком» [14. Р. 112], и предлагают ряд инициатив, которые позволили бы повысить уровень доступности публикаций, написанных на других языках (включая, например, «перестройку» практикуемого журналами регламента, которая могла бы создать новый тренд, поддерживающий многоязычную публикационную политику). Такие инициативы, по мнению авторов, позволили бы элиминировать языковые барьеры, препятствующие продуктивной научной коммуникации, в то время 
как игнорирование сложившейся тенденции приведет к тому, что значительная часть важных научных изысканий окажется «потерянной» (в терминологии авторов - «lost science»).

Однако статистика показывает, что ученые по всему миру все чаще делают выбор в пользу публикации своих исследований на английском языке, в том числе вследствие большей вероятности быть процитированными своими коллегами. Так, в 2005 г. количество англоязычных рецензируемых публикаций составляло 1,1 миллиона статей, и эта цифра увеличивается на 4\% ежегодно [15]. Активно развивающаяся индустрия онлайн-журналов стимулирует приоритетность английского языка, и именно такие журналы предоставляют авторам наилучшую перспективу для повсеместного распространения и международного признания. Это привело к тому, что количество статей, публикующихся на английском языке не англоговорящими авторами, превышает количество статей, публикуемых носителями языка [16].

По итогам сплошной выборки материал исследования представлен 26 примерами научных статей, оформленных в формате публикаций с периферийной актуальностью темы исследования. Все рассмотренные научные публикации оформлены в строгом композиционном соответствии традиционным требованиям структурирования научного текста: каждая из анализируемых статей имеет заголовок, аннотацию, ключевые слова, тематические подзаголовки (включая введение, методы исследования, заключение и т.п.) и список использованных источников (литературы). Кроме того, каждой статье присвоен идентификационный номер DOI, что упрощает поиск информации по публикациям, включая количество цитирований.

Методология оценки актуальности темы исследования основана на оценке целесообразности научной работы по следующим параметрам: теоретическая значимость темы исследования, практическая значимость темы исследования, потенциал решения конкретной общезначимой и/или научной проблемы при разработке данной темы исследования. Объективная оценка актуальности темы исследования может быть также осуществлена путем статистического анализа встречаемости данной проблематики в научной литературе, однако данный подход не применяется в настоящей работе.

Детальное ознакомление с текстами исследуемых научных публикаций позволило выделить три ключевые особенности рассматриваемого формата научных публикаций:

- сохранение познавательной функции научного текста;

- реализация развлекательной функции;

- наличие большего потенциала для самоидентификации автора научного текста.

Несмотря на специфичность формата публикаций с периферийной актуальностью темы исследования, их научно-исследовательская составляющая остается приоритетной, вследствие чего реализация познавательной 
функции в такого рода статьях не теряет своей значимости. Рассмотренная выше статья служит хорошим примером познавательной функции научной публикации с периферийной актуальностью темы исследования, так как, исследуя очевидно не первостепенную тему музыкальных предпочтений поклонников группы AC/DC, автор последовательно раскрывает методологические особенности применения микроэкономической модели ограниченной рациональности, иллюстрируя возможности исследования данной концепции через практику использования экономической экспериментальной игры «ультиматум». В результате автор приходит к выводам, имеющим опосредованное практическое значение, так как сама постановка цели исследования изначально формулируется в демонстративно иррациональной с научной точки зрения форме. С дискурсивной точки зрения, четкое следование правилам построения научной мысли, а также использование строго конвенциональных языковых средств для ее описания наделяют исследование очевидной теоретической значимостью. Как следствие, создается своего рода эффект парадокса, благодаря которому научный текст одновременно теряет и обретает значимость в зависимости от установки читателя, который может рассматривать научную публикацию как неактуальную в свете неактуальности непосредственной темы исследования или же расценивать ее как релевантный вклад в теоретическое осмысление методологии описания экономических проблем.

Рассматривая непосредственные инструменты достижения эффекта парадокса, необходимо отметить явное преобладание традиционных языковых маркеров, даже поверхностный анализ которых позволяет отнести подобные публикации к категории текстов научного дискурса. К числу таких маркеров относятся, например:

- маркеры, обозначающие начало темы:

Since 1980, there has been near constant contention regarding who was the better singer. In this paper, we explore this issue;

- маркеры, указывающие на завершение темы:

Our analysis suggests that in terms of affecting efficient decision making among listeners, Brian Johnson was a better singer;

- маркеры, обозначающие порядок следования информации в теме:

Prior to learning their roles of proposers or responders, each participant provided the offer they would extend were they assigned the role of proposer and, for each possible offer (i.e. for each offer between zero and \$10), whether they would accept or reject the offer were they assigned the role of responder. After all individuals had provided this information, the roles of proposer and responder were randomly assigned within each pair and the indicated offer (from the proposer) and the respective accept or reject decision (from the responder) were implemented;

- маркеры, обозначающие причинно-следственные отношения:

As suggested by our analysis above, we observed a higher rate of rejection and hence less efficient outcomes when the music of Bon Scott was played during participants' decision-making; 
- маркеры, отсылающие к отдельным фрагментам в тексте статьи:

Given all this, it is no wonder that AC/DC has such a rabid fan base and, as discussed below, faces an epic debate regarding its line-up;

- маркеры, отсылающие к работам других исследователей:

As demonstrated by Bernardi et al. (2006), different musical styles can have different physiological effects in individuals.

Таким образом, эффект парадокса в научных публикациях рассматриваемого формата основывается на возможности балансирования двух противоречащих друг другу компонентов - периферийной актуальности темы исследования и методологического потенциала научной работы, обеспечивающего ее познавательную значимость. Согласно проведенному анализу подобные публикации зачастую содержат информацию, исключительно полезную для представителей научного сообщества, находящихся в поиске новых исследовательских инструментов. Так, например, статья под названием «A diamond is forever» and other fairy tales: the relationship between wedding expenses and marriage duration» [17] исследует вопрос взаимосвязи между продолжительностью брака и затратами на обручальные кольца:

In this study, we evaluate the association between wedding spending and marriage duration using data from a survey of more than 3,000 ever-married persons in the United States. Controlling for a number of demographic and relationship characteristics, we find evidence that marriage duration is inversely associated with spending on the engagement ring and wedding ceremony [Ibid. P. 1919].

В настоящем исследовании рассматривается связь между затратами на свадьбу и продолжительностью брака с использованием данных, полученных в результате опроса 3000 резидентов США, когда-либо состоявших в браке. Прослеживая ряд демографических и межличностных параметров, мы находим подтверждение тому, что продолжстельность брака обратно пропорииональна затратам на обручальное кольцо и свадебную иеремонию.

Анализируя периферийную с точки зрения экономического анализа проблематику, статья одновременно несет весомый познавательный потенциал, который раскрывается через описание используемых авторами инструментов опроса, таких, например, как программная утилита Qualtrics, позволяющая осуществлять сбор и анализ данных для исследования рынка, оценки удовлетворенности и лояльности клиентов, а также тестирования продуктов и концептов, или онлайн-сервис Mechanical Turk (mTurk), который все чаще используется учеными для проведения экспериментальных исследований и анкетирования.

Кроме того, авторы предлагают вниманию читателя детальный протокол методологии анализа, который может быть применен при исследовании проблем взаимообусловленности событий и явлений. Например, к числу ключевых пунктов такого протокола, обнаруживаемых в рассматриваемой статье, относится применяемая авторами методология опроса, охватывающая обширный диапазон данных, включая информацию о текущем 
семейном положении, продолжительности брака, наличии детей, затратах на обручальные кольца, количестве приглашенных на свадьбу гостей, общих затратах на подготовку к свадебной церемонии, текущем возрасте респондента и возрасте на время замужества, этнической принадлежности, образовании, профессиональной занятости, семейном доходе, регионе проживания, вероисповедании, разнице в возрасте, этнической принадлежности и уровне образования. Экономические модели, используемые в процессе исследования, разумеется, также играют важную роль в процессе построения аргумента, доказывающего, что количество денег, затраченных на свадьбу, влияет на долговечность брачных уз. Так, в статье предлагается детальное объяснение принципа применения модели пропорциональных рисков Кокса и диагностический тест с использованием остатков Шёнфельда.

Другой особенностью научных публикаций рассматриваемого формата является реализация развлекательной функции, которая (в силу противоречия стандартам научной риторики) позволяет автору акцентировать периферийность актуальности темы исследования посредством подчеркнуто комической ее трактовки и, соответственно, обеспечить более выраженный контраст с актуальностью используемой в работе методологии.

Реализация данной функции может осуществляться посредством как языковых, так и риторических инструментов. К числу наиболее интересных примеров риторических средств реализации развлекательной функции, обнаруженных в материалах исследования, можно отнести прецедентные референции, представляющие собой «общеизвестные или известные большинству лингвокультурного сообщества единицы, хранящиеся в коллективной памяти этого сообщества, регулярно актуализирующиеся в речи, отражающие нечто фактическое, существовавшее и/или существующее в реальности и имеющие словесное выражение» [18. С. 14-15]. Согласно В.В. Красных прецедент представляет собой «элемент культуры, служащий основой для построения инвариантного восприятия, которое, в свою очередь, всегда вмещает в себя некий социокультурный опыт, консервирующийся и передающийся через воспроизводимость такого восприятия» [19. С. 70]. Другими словами, прецедент всегда исторически обусловлен и выступает в качестве сравнительной модели, с которой сопоставляется иное схожее, инвариантное знание, поведение, явление, решение и т.д.

Использование прецедентной референции при реализации развлекательной функции в научных публикациях исследуемого формата может быть проиллюстрировано на примере статьи «An option value problem from Seinfeld»[20], отсылающей к известному американскому телесериалу в жанре комедии положений «Сайнфелд», который транслировался на американском телевидении с 1989 по 1998 год. Создатели сериала Джерри Сайнфелд и Ларри Дэвид характеризовали сериал как «шоу ни о чём» («а show about nothing»), и именно данный слоган был использован автором научной статьи при составлении более чем краткой аннотации, сформулированной следующим образом: 
This is a paper about nothing [20. P. 563].

Это статья ни о чем.

Очевидно, что в данном случае особое значение имеет осведомленность читателя об оригинальном источнике, послужившем основой для эксцентричного описания научной работы. В этом смысле важным дополнением к определению прецедентных единиц становится введение параметра ассоциативности, определяющего саму природу такого рода референций. Г.Г. Слышкин предлагает рассматривать данные единицы как «целостные, образные, ассоциативные и часто экспрессивные элементы, связанные в сознании реципиентов информации с известными им текстами, ценностная значимость которых может быть достоверно установлена для определенной культурной группы» [21. С. 28].

Важность ассоциативности как элемента культурного наследия подчеркивается в гипотезе К.К. Касьяновой, которая высказывает мнение о том, что ядро этнического характера составляет некоторый набор предметов или идей, ассоциируемых в сознании каждого носителя определенной культуры с отчетливой и яркой палитрой эмоций и чувств, собирательно называемых сантиментами. Попадая в сознание реципиента, такой предмет или идея приводят в движение ассоциативные эмоциональные переживания, порождая в конечном счете более или менее типичное (но в большинстве случаев ожидаемое) действие. Таким образом формируется своего рода цепочка взаимосвязанных звеньев, где всегда присутствуют триггер (предмет) и ассоциативно обусловленное последствие (действие) [22. Р. 32]. В случае анализируемого примера в качестве последствия рассматривается реализация развлекательной функции через ассоциативное восприятие читателя, основывающееся на его знаниях и опыте, причем результирующая комичность обусловливается тем фактом, что необходимые для верного восприятия информации знания не имеют отношения к профессиональному опыту реципиента, но отсылают к его повседневной культурно-маркированной осведомленности об окружающем мире.

Языковые средства, используемые с целью реализации развлекательной функции и достижения комического эффекта, достаточно подробно изучены в работах как российских, так и зарубежных исследователей. Однако один и тот же набор языковых средств, предназначенных для создания комического эффекта, подразумевает разную интерпретацию в рамках различных типов дискурса, и в случае научного дискурса любая попытка развлечь читателя будет толковаться как намеренная генерация конфликта с дискурсивными канонами научного письма.

Так, например, статья под названием «Up or down? A male economist's manifesto on the toilet seat etiquette» [23] привлекает внимание читателя не только спецификой темы исследования (периферийной даже по самым объективным оценкам), но и выбором языковых средств, позволяющих автору реализовать нетипичную для научного дискурса развлекательную функцию:

This paper develops an economic analysis of the toilet seat etiquette. I investigate whether there is any efficiency justification for the presumption that men 
should leave the toilet seat down after use. I find that the "down rule»" (неологизм) is inefficient unless there is a large asymmetry in the inconvenience costs of shifting the position of the toilet seat across genders. I show that the "selfish» or the "status quо» (неологизм) rule that leaves the toilet seat in the position used dominates the down rule in a wide range of parameter spaces including the case where the inconvenience costs are the same [23. P. 303].

В настоящей статье предлагается экономический анализ этикетных правил пользования туалетным сидением. Я рассматриваю правомерность предположения о том, что мужчины должны опускать сиденье унитаза после использования туалета и доказываю, что «правило опускания〉 оказывается несостоятельным за исключением случаев масштабной асимметрии в неудобствах, причиняемьх в результате позиционирования туалетного сидения представителями двух полов. Я также иллюстрирую, что «правило эгоизма» или «правило статуса кво», подразумевающее, что сиденье оставляется в использованной позиции, доминирует в сравнении с правилом опускания в иироком диапазоне параметрических пространств, включая случаи тождественности причиняемых неудобств.

Непосредственным инструментом в реализации развлекательной функции выступает формулировка «toilet seat etiquette»- одновременно легитимная (учитывая, что именно данный аспект повседневной бытовой активности рассматривается в качестве ключевого предмета исследования) и ироничная (с учетом стилистического регистра, в рамках которого любая попытка насмешливой эксплуатации научной мысли неизбежно подчеркнет дискурсивное несоответствие языка и риторики научного повествования). Конфликтность как средство реализации развлекательной функции особо ярко выражается в тексте научной статьи посредством систематического использования научной терминологии, включая такие выражения, как «efficiency justification», «large asymmetry», «inconvenience costs», «across genders», «parameter spaces» и др., а также неологизмов, таких как «down rule», «selfish rule» и «status quo rule», которые, в свою очередь, приобретают исключительно специфичное (комичное) значение в контексте исследования, подчеркивая периферийность его темы. Другими словами, как и в случае с познавательной функцией, результатом подобных языковых практик является эффект парадокса, который одновременно включает и исключает текст такого рода из категории полноправных текстов научного дискурса.

Последняя особенность научных статей данного формата, рассматриваемая в настоящей работе, подразумевает наличие большего потенциала для самоидентификации автора научного текста. Поскольку статьи с периферийной актуальностью темы исследования во многих смыслах выходят за рамки нормативных стандартов научного дискурса, некоторые фиксированные правила, такие как сдержанность и дистанцированность автора, могут отходить на второй план, позволяя исследователю «приблизиться» к читателю и идентифицировать себя через языковые средства, нетипичные для научной риторики. По результатам проведенного анализа, идентифи- 
цирующая функция реализуется в том или ином виде в приблизительно 77\% случаев, т.е. 20 из 26 проанализированных статей содержали дискурсивные элементы, позволившие авторам идентифицировать себя в тексте работы. Стоит отметить, что с учетом традиционно принятого регламента научного дискурса, постулирующего обособленность и максимальную дистанцированность исследователя с целью обеспечения максимальной объективности, распространенность инструментов языковой самоидентификации в рассматриваемых статьях в очередной раз свидетельствует об их особенной специфике, поскольку они позволяют автору экспериментировать с дискурсивными практиками без ущерба для научно-исследо-вательской составляющей работы в целом. При этом «формула успеха» во многом определяется именно отсутствием необходимости в объективной оценке фактов, так как сама тема исследования изначально периферийна (минимально актуальна) по своей природе. Это открывает простор для творчества, давая автору возможность раскрыть научный потенциал исследования, одновременно сблизившись с читателем через самоидентификацию.

Самоидентификация автора в статьях рассматриваемого формата в первую очередь реализуется через актуализацию оппозиции «свой - чужой» посредством акцентирования институциональной и профессиональной связи между автором и читателем как представителей научного сообщества. Ярким примером, иллюстрирующим данное положение, служит статья «The whimsical science» [24], причем в данном случае один только заголовок научной публикации («Причудливая наука») уже несет в себе идентифицирующую функцию вне зависимости от содержания статьи, и осознать этот потенциал сможет только член соответствующего (в данном случае - экономического) профессионального сообщества, по роду деятельности знакомый с термином «the dismal science». «The dismal science» («мрачная наука») - уничижительное альтернативное название для экономики, впервые предложенное в XIX в. викторианским историком Томасом Карлайлом для акцентирования присущего ей фатализма, а также в качестве контраста с распространенным в то время термином «gay science» («веселая наука»), отсылавшим к практикам сочинения песен и стихов. Обладая данным знанием, читатель имеет возможность расшифровать языковой оборот, превративший устоявшийся термин в эксцентричный каламбур и позволивший автору идентифицировать себя как «своего», т.е. члена профессионального сообщества.

Как видно из данного примера, возможность самоидентификации в статьях рассматриваемого формата может быть связана с наличием развлекательного компонента, и такая взаимосвязь четко прослеживается в анализируемом материале. Например, содержание статьи «Your right arm for a publication in AER?» [25] раскрывается авторами в аннотации следующим образом:

The time tradeoff (TTO) method is popular in medical decision making for valuing health states. We use it to elicit economists' preferences for publishing in top economic journals and for living without limbs. The economists value journal publi- 
cations highly and have a clear preference among them, with the American Economic Review (AER) the most preferred. Their responses imply they would sacrifice more than half a thumb for an AER publication. These TTO results are consistent with ranking and willingness to pay results, and indicate that journal preferences are not entirely determined by impact factors or by expectations of a salary increase following a publication in a prestigious journal [25. P. 495].

Метод временного компромисса (МВК) популярен в принятии медицинских решений для оценки состояния здоровья. Мы используем данный метод для выявления предпочтений экономистов касательно публикачии научных статей в ведущих экономических журналах, а также в отношении жизни без конечностей. Экономисты высоко ценят публикации в журналах и имеют явные предпочтения среди них, причем наиболее востребованным является журнал American Economic Review (AER). Согласно ответам респондентов они согласились бы пожертвовать более половиной пальца, если бы это позволило им опубликоваться в AER. Результать МВК-анализа согласуются с данныли, отражающими взаимосвязь между рейтингом журнала и готовностью к пожертвованиям, а также доказывают, что выбор журнала не полностью определяется импакт-фактором или ожиданием повышенной зарплаты в результате публикации статьи в престижном журнале.

Помимо очевидной развлекательной составляющей, заставляющей читателя задуматься над тем, сколько конечностей он готов пожертвовать ради публикации в престижном научном издании, данная статья не менее очевидным образом идентифицирует автора как человека, близко знакомого с современными требованиями публикационной активности, предъявляемыми к преподавателям и ученым. При этом сочетание развлекательной и идентифицирующей функций дает автору возможность сблизиться с читателем на новом уровне, где наука, повседневность и чувство юмора «сплачиваются», генерируя новый формат площадки для научной дискуссии.

Рассмотренные выше примеры, а также большинство статей, послуживших материалом для настоящего исследования, указывают на другой типичный способ реализации идентифицирующей функции, актуализируемый в статьях с периферийной актуальностью темы исследования, а именно использование местоимения первого лица единственного и множественного числа как в аннотациях, так и в тексте статьи. С точки зрения традиционной лингвистики текста «я» и «мы» рассматриваются как средства реализации личностной референции, «обозначающие личность и ее роль в контексте речевой ситуации» [26. Р. 263]. Использование местоимений «я» или «мы» позволяет эксплицировать информацию в рамках лингвистической экспоненты «автор-знание», т.е. «Я / мы полагаем / утверждаем / считаем...» и т.п. Однако с точки зрения реализации дискурса, в том числе научного, данные местоимения смещают «ось» текстовой информации в сторону межличностной экспоненты «автор - читатель» [27]. Иными словами, функционируя в составе дискурсивных практик обмена информацией, местоимения «я» и «мы» отражают отношения вне границ семан- 
тики текста как такового, но в рамках более обширной области социальных ролей и взаимоотношений.

Стоит отметить, что исследование дискурсивных особенностей употребления местоимения первого лица единственного и множественного числа требует сужения рамок анализа до определенных типов институционального дискурса, так как разные типы институционального дискурса могут «наделять» данное местоимение разнородными функциями, подразумевающими включение или, наоборот, отстранение референта от текста и целевой аудитории. Так, например, по замечанию М. Халлидея и Р. Хасан [27. Р. 53], местоимение «мы», используемое врачом в рамках медицинского дискурса, может рассматриваться как стратегия кооперации (например, «How are we feeling today?»), в то время как в рамках публицистического дискурса то же местоимение позволит автору говорить одновременно за себя и за редколлегию, подчеркивая единство людей, сплоченных коллективными интересами [28].

Анализируя использование местоимений «я» и «мы» в научном дискурсе, необходимо в первую очередь обозначить специфику их функционирования именно в англоязычных научных текстах. Согласно сравнительному исследованию, проведенному И. Вассилевой [29], корпусный анализ значительного объема данных свидетельствует об очевидно более выраженной тенденции к использованию этих местоимений в англоязычных публикациях по сравнению с публикациями, написанными на немецком, французском, русском и болгарском языках:

- местоимение «я»: 69\% в английском, 47\% в немецком, 40\% во французском, $0,5 \%$ в русском, $6 \%$ в болгарском;

- местоимение «мы»: 31\% в английском, 53\% в немецком, 60\% во французском, 95,5\% в русском, 94\% в болгарском [Ibid. P. 165].

При этом автор приходит к выводу, что ключевая функция, реализуемая при выражении «авторского присутствия» («author presence») посредством использования местоимения первого лица единственного или множественного числа, заключается в определении границ «авторской ответственности» («author responsibility»), когда местоимение «я» позволяет исследователю позиционировать себя через дискурс в качестве единственного автоpa, ответственного за содержание статьи, в то время как местоимение «мы» служит индикатором коллективной мысли и одновременно инструментом отрицания соревновательного подхода [Ibidem].

Анализ данной проблемы в контексте исследования англоязычных научных публикаций с периферийной актуальностью темы исследования показал наличие устойчивой и преобладающей тенденции к использованию местоимений «я» и «мы», что можно проследить на примере материалов, рассмотренных выше в настоящей статье. Однако в качестве основной причины или мотива выражения авторского присутствия в публикациях рассматриваемого формата выступает, на наш взгляд, не параметр «ответственности», а стремление автора к самоидентификации и сближению с читателем, что становится возможным благодаря установлению менее 
строгих рамок научной риторики. Наиболее ярким примером может служить статья «Suitable research: on the development of a positive theory of the business suit» [30], в которой местоимения «я» и «мы» используются последовательно на протяжении всего текста научной публикации, посвященной изучению переменных, обусловливающих выбор делового костюма молодыми бизнесменами, а также экономических предпосылок и последствий модных пристрастий, встречающихся в деловых кругах:

For years I have been fascinated by the phenomenon of the business suit. Why is a suit the prevalent attire for business people? Why is it especially predominant among accountants and auditors? Can we explain the variations in color, cut, number of pieces, etc., that we so readily observe? Can we predict how the choice of a business suit will be affected by changes in the underlying variables? If and when constructed, a Positive Theory of the Business Suit will allow us to explain and predict these phenomena.

Some research has been conducted in this area. However, you will agree with me that previous studies have been predominantly normative and that the economic viewpoint has been relatively neglected. Yet, it is evident that this is an area where real economic costs are involved. What is more, these costs are borne in the first instance by the economic actors themselves. We therefore need an economic theory to explain why young executives already choose to wear a suit when they are just entering the firm. Hence, I posit such a theory based upon the selfinterest of the actors and I show that (expected) economic benefits are involved as well. Furthermore, I indicate how these costs and benefits change as the executive climbs the organizational ladder and I predict how this will affect the choice of his suit. In particular, I show how the cost burden gradually shifts towards the firm transforming the business suit from a private cost to a corporate perquisite. For the top executives, I show how potential conflicts of interest may thus arise between them and their principals, the shareholders, and how these conflicts will be resolved in the employment contracts [Ibid. P. 105].

Рассмотренный фрагмент научного текста отмечен использованием местоимения «я» в качестве не столько предпочтительного или превалирующего, но единственно доминирующего способа языковой идентификации автора, причем в качестве отдельной дискурсивной особенности в данном случае можно выделить намеренно повторяющееся применение конструкций, начинающихся с «я» (анафора), таких как «я утверждаю», «я показываю», «я иллюстрирую» и «я предсказываю», что особенно нехарактерно для традиционных статей как элементов научного дискурса. Местоимение «мы» служит инструментом сближения автора и читателя, идентифицируя их как заинтересованных лиц, объединенных общими стремлениями и задающихся одинаковыми исследовательскими вопросами. Как можно заметить, автор также прибегает к прямой апелляции к читателю через местоимение «вы» («уоu will agree with mе»), что может рассматриваться как еще более настойчивая попытка к сближению с читателем.

Наконец, идентифицирующая функция в англоязычных научных публикациях с периферийной актуальностью темы исследования находит про- 
дуктивное выражение через упомянутые ранее в статье прецедентные референции, посредством которых автор позиционирует себя в качестве не отдельного индивида, а представителя социокультурного сообщества, объединяющего его с читателем. В данном контексте на передний план выходят положения теории, выдвинутой Д.Б. Гудковым [31], который выделил несколько уровней прецедентности, формирующей сознание индивида:

- автопрецедентные референции порождают ассоциативные ряды, эмоции и представления сугубо личного характера и в первую очередь связаны с опытом, воспоминаниями, хронологией, людьми, событиями и знаниями, которые попадают под определение неповторимого, индивидуального опытного и эмоционального «багажа», связанного в сознании реципиента с соответствующими концептами реальной действительности. Например, проводя мысленную ассоциативную аналогию при анализе прецедента, осуществляющего отсылку к стеклянному стакану, результирующий образ в сознании разных людей может быть реализован в виде газировки из автомата, крепкого алкогольного напитка, памятного сувенира, продукции стекольного завода и т.д. Другими словами, вероятность вариации ассоциативного значения, порождаемого данным видом прецедента у разных индивидов, оказывается очень высокой в силу различия имеющегося у людей опыта;

- социумно-прецедентные референции представляют собой отсылки, в той или иной степени известные представителю того или иного социума и являющиеся частью так называемого «коллективного когнитивного пространства». Это пространство может быть ограничено, например, рамками религиозного, профессионального, семейного и других видов социума. Так, библейские тексты будут считаться отчетливо прецедентными для представителей религиозного социума, а теория моделирования экономических процессов станет прецедентом для специалистов в области эконометрики (профессиональный социум) и т.д. [32];

- национально-прецедентные референции расширяют рамки результирующих ассоциативных представлений еще больше, входя в когнитивную базу целого лингвокультурного сообщества. В данном случае вероятность вариации ассоциативного значения, порождаемого у разных индивидов, значительно снижается, так как такие прецеденты оказываются знакомыми для более широких групп людей;

- универсально-прецедентные референции известны большинству людей и входят в универсальное когнитивное пространство человечества. Другими словами, вероятность узнаваемости универсально-прецедентного феномена может быть описана как наиболее высокая.

Таким образом, используя прецедентную референцию, автор получает возможность идентифицировать себя в рамках двух «узких» областей коллективного позиционирования - социумно-прецедентного и национальнопрецедентного, в то время как автопрецедентные и универсально-прецедентные референции стали бы маркерами эгоцентрированного или, соответственно, чрезмерно генерализированного дискурсивного оформления 
научного текста. В то же время сама возможность раскрытия социокультурной идентичности автора в рамках научного дискурса в очередной раз указывает на уникальность подобного рода публикаций как специфичного формата научного письма.

Необходимо отметить, что, хотя анализируемый феномен не может рассматриваться в качестве масштабного явления, статьи подобного формата представляют собой интересный пример нетрадиционных дискурсивных практик и могут стать предметом более детального изучения в научной литературе.

\section{Литература}

1. Матвеева T.B. Полный словарь лингвистических терминов. Ростов н/Д : Феникс, 2010. $562 \mathrm{c}$.

2. Кравцева E.B. Научный дискурс как вид институционального типа дискурса // Вестник ЮУрГУ. 2012. № 25. С. 130-131.

3. Шаменкова Е.А. Исследовательская деятельность: словарь. М. : Перспектива, 2010. $88 \mathrm{c}$.

4. Oxoby R.J. On the efficiency of AC/DC: Bon Scott versus Brian Johnson // Economic Inquiry. 2009. № 47 (3). P. 598-602. DOI: 10.1111/j.1465-7295.2008.00138.x

5. Карчаева С.Х. Дискурсивность научного текста : автореф. дис. ... канд. филол. наук. Нальчик, 2010. 24 с. URL: https:/dspace.kpfu.ru/xmlui/bitstream/handle/net/ 112347/0-786116.pdf?sequence=1 (дата обращения: 29.07.2018).

6. Ивин А.А. Современная философия науки. М. : Directmedia, 2015. 838 с.

7. Болдырева А.А., Кашкин В.Б. Категория авторитетности в научном дискурсе // Язык, коммуникация и социальная среда. 2006. № 1. С. 58-70.

8. Семененко И.С. Нация, национализм, национальная идентичность: новые ракурсы научного дискурса // Мировая экономика и международные отношения. 2015. № 59 (11). C. 91-102.

9. Hamilton C.E., Carter-Thomas S. Competing influences: the impact of mode and language on verb type and density in French and English scientific discourse // CHIMERA: Romance Corpora and Linguistic Studies. 2016. № 4 (1). P. 13-34.

10. Long K.P. Gender and scientific discourse in early modern culture. Routledge, 2016. $312 \mathrm{p}$.

11. Dorgeloh H., Wanner A. Formulaic argumentation in scientific discourse // Formulaic Language. 2009. № 83. P. 523.

12. Shehzad $W$. Explicit author in scientific discourse: a corpus-based study of the author’s voice // Malaysian Journal of ELT Research. 2016. № 3 (1). P. 18.

13. Livnat $Z$. Impersonality and grammatical metaphors in scientific discourse. The rhetorical perspective // Lidil. Revue de linguistique et de didactique des langues. 2010. № 41. P. 103-119.

14. Meneghini R., Packer A.L. Is there science beyond English? Initiatives to increase the quality and visibility of non-English publications might help to break down language barriers in scientific communication // EMBO reports. 2007. № 8 (2). P. 112-116. DOI: 10.1038/sj.embor.7400906

15. Kulczycki E., Engels T.C., Pölönen J., Bruun K., Dušková M., Guns R., Zuccala A. Publication patterns in the social sciences and humanities: evidence from eight European countries // Scientometrics. 2018. № 1 (116). P. 463-486.

16. Flowerdew J. Some thoughts on English for research publication purposes (ERPP) and related issues // Language Teaching. 2015. № 48 (2). P. 250-262. 
17. Francis-Tan A., Mialon H.M. «A diamond is forever» and other fairy tales: the relationship between wedding expenses and marriage duration // Economic Inquiry. 2015. № 53 (4). P. 1919-1930. DOI: 10.1111/ecin.12206

18. Гудков Д.Б. Теория и практика межкультурной коммуникации. М. : ИТДГК Гнозис, 2003. 288 с.

19. Kpacных B.B. Когнитивная база и прецедентные феномены в системе других единиц и в коммуникации // Вестник МГУ. 1997. № 9 (3). С. 62-75.

20. Dixit A. An option value problem from Seinfeld // Economic Inquiry. 2012. № 50 (2). P. 563-565. DOI: 10.1111/j.1465-7295.2011.00377.x

21. Слышкин Г.Г. От текста к символу: лингвокультурные концепты прецедентных текстов в сознании и дискурсе. М. : Academia, 2000. 128 с.

22. Malyuga E., McCarthy M. English and Russian vague category markers in business discourse: linguistic identity aspects // Journal of Pragmatics. 2018. № 135. P. 39-52. DOI: 10.1016/j.pragma.2018.07.011

23. Choi J.P. Up or down? A male economist's manifesto on the toilet seat etiquette // Economic Inquiry. 2011. № 49 (1). P. 303-309. DOI: 10.1111/j.1465-7295.2009.00277.x

24. Levins R.A. The whimsical science // Applied Economic Perspectives and Policy. 1992. № 14 (1). P. 139-151. DOI: 10.2307/1349614

25. Attema A.E., Brouwer W.B.F., Van Exel J. Your right arm for a publication in AER? // Economic Inquiry. 2014. № 52 (1). P. 495-502. DOI: 10.1111/ecin.12013

26. Cherry R.D. Ethos versus persona: self-representation in written discourse // Written communication. 1988. № 5 (3). P. 251-276.

27. Halliday M.A.K., Hasan R. Cohesion in English. London : Longman, 1976. 375 p.

28. Smirnova A. Argumentative use of reported speech in British newspaper discourse // Text \& Talk. 2012. № 32 (2). P. 235-253.

29. Vassileva I. Who am I/who are we in academic writing? A contrastive analysis of authorial presence in English, German, French, Russian and Bulgarian // International Journal of Applied Linguistics. 1998. № 8 (2). P. 163-190.

30. Schreuder $H$. Suitable research: on the development of a positive theory of the business suit // Accounting, Organizations and Society. 1985. № 10 (1). P. 105-108. DOI: 10.1016/0361-3682(85)90034-0

31. Гудков Д.Б. Прецедентные феномены в языковом сознании и межкультурной коммуникации : дис. ... д-ра филол. наук. М., 1999. 400 с.

32. Malyuga E.N., Krouglov A., Tomalin B. Linguo-cultural competence as a cornerstone of translators' performance in the domain of intercultural business communication // xLinguae. 2018. № 11 (2). P. 566-582. DOI: 10.18355/XL.2018.11.02.46

\section{Emergent Trends in English Scientific Discourse: Issues of Research Relevance and Linguistic Identity}

Vestnik Tomskogo gosudarstvennogo universiteta. Filologiya - Tomsk State University Journal of Philology. 2019. 58. 52-70. DOI: 10.17223/19986645/58/4

Elena N. Malyuga, RUDN University (Peoples' Friendship University of Russia) (Moscow, Russian Federation). E-mail: malyuga_en@rudn.ru

Keywords: scientific discourse, identity, scientific publication, scientific article, relevance of research, discourse marker, identifying function, precedent.

The publication was prepared with the support of the "RUDN University Program 5-100".

The object of this study is English scientific discourse with a scientific text viewed as its unit. The subject of the study is represented by topic relevance and research methodology as parameters of a scientific text. The hypothesis of the study postulates that modern English scientific discourse has accommodated a clearly identifiable unconventional format for the construction of scientific rhetoric. The essence of this format is that topic relevance is 
deliberately assigned a peripheral role, while research methodology, on the contrary, comes to the foreground, thus turning into the key goal of the scientific endeavor in general. This format is characterized as unique, inasmuch as it incorporates two interrelated and clearly contrasting components of a scientific text - the "peripheral" relevance of the topic of a study and priority ranking of its methodology. The key aim of the present article is to analyze the discursive peculiarities of this type of scientific texts in English scientific discourse. The material for the study is represented by English research articles on economics published in high-rated peer-reviewed journals. The methodology for assessing topic relevance implied assessing its feasibility against the following parameters: its theoretical significance; its practical significance; and the potential to address a specific, generally valid and/or scientific problem by exploring this topic. As a result of the study, the author identifies three peculiarities of this kind of articles: preservation of the gnostic function, realization of the entertaining function, and better potential for author's self-identification. The specifics of the discursive aspects of the format under discussion is described through the terminology used, the phenomenon of precedence, discursive markers, the use of the pronoun "I". The author concludes that with this type of format in English scientific discourse the scientific text simultaneously loses and acquires significance depending on the reader's appraisal, whereby the reader can view the scientific publication as irrelevant in the light of the irrelevance of the immediate topic of research, or regard it as a relevant contribution to the theoretical comprehension of the methodology to be used in addressing scientific issues. The author also postulates that although this phenomenon does not acquire massive proportions, articles with peripheral relevance of research topic can be considered an interesting example of nontraditional discursive practices and can become subject of more detailed research in the future.

\section{References}

1. Matveeva, T.V. (2010) Polnyy slovar' lingvisticheskikh terminov [A comprehensive dictionary of linguistic terms]. Rostov-on-Don: Feniks.

2. Kravtsova, E.V. (2012) Research discourse as type of institutional discourse. Vestnik YuUrGU-Bulletin of the South Ural State Universitety. 25. pp. 130-131. (In Russian).

3. Shashenkova, E.A. (2010) Issledovatel'skaya deyatel'nost': slovar' [Research activites: a dictionary]. Moscow: Perspektiva.

4. Oxoby, R.J. (2009) On the efficiency of AC/DC: Bon Scott versus Brian Johnson. Economic Inquiry. 47 (3). pp. 598-602. DOI: 10.1111/j.1465-7295.2008.00138.x

5. Karchaeva, S.Kh. (2010) Diskursivnost' nauchnogo teksta [Discursiveness of a research text]. Abstract of Philology Cand. Diss. Nalchik. [Online] Available from: https://dspace.kpfu.ru/xmlui/bitstream/handle/net/112347/0-786116.pdf?sequence=1.

(Accessed: 29.07.2018).

6. Ivin, A.A. (2015) Sovremennaya filosofiya nauki [Modern philosophy of science]. Moscow: Directmedia.

7. Boldyreva, A.A. \& Kashkin, V.B. (2006) Kategoriya avtoritetnosti v nauchnom diskurse [The category of authority in academic discourse]. Yazyk, kommunikatsiya $i$ sotsial'naya sreda - Language, Communication and Social Environment. 1. pp. 58-70.

8. Semenenko, I.S. (2015) Nations, Nationalism, National Identity: New Dimensions in Academic Discourse. Mirovaya ekonomika i mezhdunarodnye otnosheniya. 59 (11). pp. 91102. (In Russian).

9. Hamilton, C.E. \& Carter-Thomas, S. (2016) Competing influences: the impact of mode and language on verb type and density in French and English scientific discourse. CHIMERA: Romance Corpora and Linguistic Studies. 4 (1). pp. 13-34.

10. Long, K.P. (2016) Gender and scientific discourse in early modern culture. Routledge.

11. Dorgeloh, H. \& Wanner, A. (2009) Formulaic argumentation in scientific discourse. Formulaic Language. 83. 
12. Shehzad, W. (2016) Explicit author in scientific discourse: a corpus-based study of the author's voice. Malaysian Journal of ELT Research. 3 (1).

13. Livnat, Z. (2010) Impersonality and grammatical metaphors in scientific discourse. The rhetorical perspective. Lidil. Revue de linguistique et de didactique des langues. (41). pp. 103-119.

14. Meneghini, R. \& Packer, A.L. (2007) Is there science beyond English? Initiatives to increase the quality and visibility of non-English publications might help to break down language barriers in scientific communication. EMBO reports. 8 (2). pp. 112-116. DOI: 10.1038/sj.embor.7400906

15. Kulczycki, E. et al. (2018) Publication patterns in the social sciences and humanities: evidence from eight European countries. Scientometrics. 1 (116). pp. 463-486.

16. Flowerdew, J. (2015) Some thoughts on English for research publication purposes (ERPP) and related issues. Language Teaching. 48 (2). pp. 250-262.

17. Francis-Tan, A. \& Mialon, H.M. (2015) "A diamond is forever" and other fairy tales: the relationship between wedding expenses and marriage duration. Economic Inquiry. 53 (4). pp. 1919-1930. DOI: 10.1111/ecin.12206

18. Gudkov, D.B. (2003) Teoriya i praktika mezhkul'turnoy kommunikatsii [Theory and practice of intercultural communication]. Moscow: ITDGK Gnozis.

19. Krasnykh, V.V. (1997) Kognitivnaya baza i pretsedentnye fenomeny v sisteme drugikh edinits i v kommunikatsii [Cognitive basis and precedent phenomena in the system of other units and in communication]. Vestnik MGU-MSU Vestnik. 9 (3). pp. 62-75.

20. Dixit, A. (2012) An option value problem from Seinfeld. Economic Inquiry. 50 (2). pp. 563-565. DOI: $10.1111 / \mathrm{j} .1465-7295.2011 .00377 . \mathrm{x}$

21. Slyshkin, G.G. (2000) Ot teksta k simvolu: lingvokul turnye kontsepty pretsedentnykh tekstov v soznanii i diskurse [From text to symbol: linguocultural concepts of precedent texts in consciousness and discourse]. Moscow: Academia.

22. Malyuga, E. \& McCarthy, M. (2018) English and Russian vague category markers in business discourse: linguistic identity aspects. Journal of Pragmatics. 135. pp. 39-52. DOI: 10.1016/j.pragma.2018.07.011

23. Choi, J.P. (2011) Up or down? A male economist's manifesto on the toilet seat etiquette. Economic Inquiry. 49 (1). pp. 303-309. DOI: 10.1111/j.1465-7295.2009.00277.x

24. Levins, R.A. (1992) The whimsical science. Applied Economic Perspectives and Policy. 14 (1). pp. 139-151. DOI: 10.2307/1349614

25. Attema, A.E., Brouwer, W.B.F. \& Van Exel, J. (2014) Your right arm for a publication in AER? Economic Inquiry. 52 (1). pp. 495-502. DOI: 10.1111/ecin.12013

26. Cherry, R.D. (1988) Ethos versus persona: self-representation in written discourse. Written Communication. 5 (3). pp. 251-276.

27. Halliday, M.A.K. \& Hasan, R. (1976) Cohesion in English. London: Longman.

28. Smirnova, A. (2012) Argumentative use of reported speech in British newspaper discourse. Text \& Talk. 32 (2). pp. 235-253.

29. Vassileva, I. (1998) Who am I/who are we in academic writing? A contrastive analysis of authorial presence in English, German, French, Russian and Bulgarian. International Journal of Applied Linguistics. 8 (2). pp. 163-190.

30. Schreuder, H. (1985) Suitable research: on the development of a positive theory of the business suit. Accounting, Organizations and Society. 10 (1). pp. 105-108. DOI: 10.1016/0361-3682(85)90034-0

31. Gudkov, D.B. (1999) Pretsedentnye fenomeny v yazykovom soznanii i mezhkul'turnoy kommunikatsii [Precedent phenomena in linguistic consciousness and intercultural communication]. Philology Dr. Diss. Moscow.

32. Malyuga, E.N., Krouglov, A. \& Tomalin, B. (2018) Linguo-cultural competence as a cornerstone of translators' performance in the domain of intercultural business communication. xLinguae. 11 (2). pp. 566-582. DOI: 10.18355/XL.2018.11.02.46 